\title{
Los Profesionales Educativos como Agentes de Capacitación Socio-Laboral de los Jóvenes con Diversidad Funcional Intelectual
}

\author{
Educational Professionals as Socio-Labor Training Agents for Youth with Intellectual Functional Diversity \\ Profissionais da Educação como Agentes de Formação Sócio-Emprego dos Jovens com Diversidade Funcional
}

María Isabel Negri Cortés* (http://orcid.org/0000-0003-0548-2187)

Departamento de Didáctica y Organización Escolar, Universidad de Málaga, España Juan José Leiva Olivencia** (http://orcid.org/0000-0002-2857-8141)

Departamento de Didáctica y Organización Escolar, Universidad de Málaga, España
Recibido: 22-02-17

Revisado: 22-05-17 Aceptado: 10-06-17

Publicado: 26-06-17
RESUMEN. La presente investigación persigue definir y comprender el papel que desempeñan los profesionales educativos como agentes responsables de la capacitación de los jóvenes con Diversidad Funcional Intelectual (en adelante DFI) en habilidades y competencias socio-laborales. Para ello, nos decantamos por un diseño de investigación mixta, utilizando cuestionarios y entrevistas semiestructuradas para la recogida de información. De esta forma, indagamos en la importancia de otorgar una formación de calidad a orientadores y docentes, que les capacite para fomentar en los jóvenes con DFI el desarrollo de habilidades y competencias sociales y laborales que faciliten su acceso al empleo y a la vida adulta. Además, descubrimos la relevancia de formar a estos profesionales en metodologías innovadoras que atiendan a la diversidad de su alumnado y promuevan la transferibilidad de los aprendizajes hacia nuevos contextos; y también la gran implicación que existe por parte de estos profesionales, aunque carezcan de los recursos suficientes.
Palabras clave: Educación y empleo, transición a la vida activa, profesionales de la educación, integración social, deficiencia mental.
ABSTRACT. This paper seeks to describe and analyze the role of professionals in the field of education as one of the main agents who contribute to the socio-labor inclusion of youth with Intellectual Functional Diversity (IFD). Based on the complementarity of the quantitative and qualitative methods, we have delved into the significance of providing
Key words: education and employment, transition to active life, 
quality training to advisors and faculty, one which will enable them to foster the development of personal, social and labor skills and competencies in youth with IFD, so they can access employment and adulthood in an easier manner. Additionally, we have highlighted the significance of training these professionals in innovative methodologies to respond to their students' diversity and promote the transferability of their learning to new contexts. To conclude, we have been aware of these professionals' great commitment despite the lack of sufficient human and material resources. educational professionals, social

integration, functional diversity.

RESUMO. Este artigo tem como objetivo descrever e analisar o papel dos profissionais na área da educação como um dos principais agentes que contribuem para a inclusão sócio-laboral dos jovens com diversidade funcional intelectual (doravante DFI). Com base na complementaridade das metodologias quantitativas e qualitativas, pesquisamos a importância de fornecer formação de qualidade aos conselheiros e professores, para que possam incentivar nos jovens com DFI o desenvolvimento de competências e habilidades pessoais, sociais e de trabalho que facilitem seu acesso ao emprego e na vida adulta. Também destacamos a importância da formação destes profissionais em metodologias inovadoras que abordem a diversidade dos seus alunos e promovam a transferência de aprendizagem em novos contextos. Finalmente, tivemos consciência do grande envolvimento

Palavraschave: educação e emprego, transição para a vida ativa, profissionais da educação, integração social, diversidade funcional. dos profissionais, apesar da falta de recursos humanos e materiais suficientes.

El término mujeres y hombres con diversidad funcional nace en el Foro de Vida Independiente del 2005, de la mano de los miembros del colectivo, que deciden autonombrarse y definirse para reivindicar su papel activo dentro de la sociedad (Rodríguez \& Ferreira, 2008). Estas personas asumen sus diferencias como un valor de enriquecimiento social, señalando que requieren de una serie de apoyos para acceder a los contextos de una sociedad que no ha tenido en cuenta sus individualidades (Romañach \& Lobato, 2007). Actualmente existe cierta controversia en relación con este cambio terminológico, que a pesar de contar con el respaldo de activistas del colectivo como Romañach y Lobato (2007) y Romañach y Arnau (2006), también despierta la discrepancia de los representantes de las diversas organizaciones estatales. Recientemente, el Comité de Entidades Representantes de Personas con Discapacidad de Canarias (CERMI Canarias) (La Opinión de Tenerife, 2017) y el Comité Catalán de Representantes de Personas con Discapacidad (COCARMI) (La Vanguardia, 2017) rechazan el uso de este término por considerarlo que es ambiguo y que podría generar confusión jurídica, reivindicando así el uso del término personas con discapacidad, que es el socialmente reconocido.

En nuestra investigación decidimos utilizar el término personas con diversidad funcional, dado que abordamos el tema desde una perspectiva pedagógica inclusiva, en cuyo enfoque consideramos fundamental escuchar y comprender las voces del colectivo, estando además de acuerdo con Rodríguez 
y Ferreira (2008), en que quien nombra es quien se considera con el poder o la autoridad suficiente para nombrar y predefinir las características del otro.

Es indudable el progreso experimentado en las últimas décadas relativo a la situación social y educativa de las personas con DFI, aunque el acceso al mercado laboral de estas parece ser todavía una asignatura pendiente. El centro educativo, como espacio reproductor de las dinámicas sociales y humanas, se constituye como una plataforma eficaz para la capacitación de sus estudiantes en habilidades y competencias personales, sociales y laborales.

Podemos encontrar varias investigaciones que ponen de manifiesto que la formación recibida en las etapas anteriores a la inclusión laboral constituye un factor clave para mejorar las oportunidades de desarrollar un rol laboral en el mercado ordinario (Pallisera, Fullana \& Vilà, 2005 como se citó en Vilà, Pallisera \& Fullana, 2010). Además, dadas las características de los jóvenes a los que hacemos referencia, así como las exigencias de un mercado laboral cada vez más dinámico y competitivo, se hace necesario comenzar la formación para el empleo durante los niveles de educación secundaria.

Otras estrategias formativas ofertadas desde el sistema educativo español, adecuadas para la capacitación en competencias de índole socio-laboral de los jóvenes con DFI son los llamados Programas de Cualificación Profesional Inicial (PCPI) en el momento de nuestra investigación, que actualmente son denominados como Ciclos de Formación Profesional Básica (FPB). Los PCPI aparecen en la Ley Orgánica de Educación (LOE, Boletín Oficianl del Estado, 2006), y son programas educativos destinados al alumnado que cuenta con una trayectoria de fracaso escolar, como es el caso de los jóvenes con DFI. El objetivo de estos programas es la capacitación laboral de su alumnado a través de la atención a la diversidad y de una metodología eminentemente práctica, que facilitarán su salida al mercado de trabajo con una titulación oficial. Recientemente, en la nueva Ley Orgánica de Mejora de la Calidad Educativa (LOMCE, Boletín Oficial del Estado, 2013), se ha modificado la organización de estos itinerarios, dando lugar a los nuevos FPB.

Durante los momentos a los que hacemos referencia, las etapas de educación secundaria y formación profesional, resulta esencial el papel desempeñado por los profesionales del ámbito escolar, en tanto que son los principales agentes para la educación y formación de este tipo de alumnado. Estos profesionales tienen un trato directo y diario con los jóvenes con DFI, lo cual les permite conocer sus capacidades, intereses y dificultades personales, así como tener un trato continuado con sus familias. Es por esta razón que nos preocupa cómo es la situación actual que viven los docentes, orientadores educativos y personal de apoyo a la integración que intervienen en la vida escolar del alumnado con DFI.

El estudio de investigación que presentamos en estas páginas forma parte de la tesis doctoral de Negri (2016), que tiene como finalidad conocer y valorar la realidad formativa y socio-laboral a la que tienen que enfrentarse los jóvenes con DFI una vez finalizan sus estudios obligatorios. En la investigación que exponemos en este artículo hemos abordado la importancia de los profesionales educativos como uno de los principales agentes para la capacitación socio-laboral de los jóvenes con DFI. Para ello, hemos definido y analizado el papel que desempeñan los profesionales del ámbito educativo durante la etapa secundaria y la formación profesional de este tipo de alumnado. 
Tomando como referencia este objetivo, nos hemos planteado otros objetivos específicos, tales como valorar la adecuación de la formación recibida por los docentes y orientadores de los centros educativos en función de su eficacia para atender a la diversidad de su alumnado y para hacer sus aprendizajes transferibles al mundo laboral. Además, hemos querido indagar en la necesidad de que estos realicen un trabajo interdisciplinar que permita la optimización los recursos del centro, y también en si es suficiente el volumen de recursos materiales y humanos que la institución educativa destina a tal fin. Por último, nos hemos propuesto establecer cuál es el grado de implicación de estos profesionales en el desempeño de sus responsabilidades.

\section{EL PAPEL DE LOS PROFESIONALES EDUCATIVOS EN LA CAPACITACIÓN SOCIO-LABORAL DE LOS JÓVENES CON DFI}

Como hemos visto, existen programas específicamente dirigidos a la formación socio-laboral de los jóvenes con DFI, los Ciclos de FPB, conocidos hasta ahora como PCPI. Estos programas, según Jurado, Olmos y Pérez (2015) pueden entenderse como una medida de promoción del desarrollo personal y de adquisición de competencias para la incorporación al mundo del trabajo, debido a su carácter especializado, su metodología práctica y su clara intencionalidad laboral, que tratan de responder a las demandas del mercado laboral y a las necesidades e intereses de su alumnado. No obstante, los profesionales del ámbito educativo deben asumir que los procesos de orientación, capacitación y formación en habilidades para el empleo y la inserción laboral no deben demorarse a la finalización de los estudios obligatorios de estos jóvenes, siendo necesario que se inicien en las etapas de educación secundaria obligatoria, tal como valoran Vilà, Pallisera y Fullana (2010). A su vez, autores como Vilà, Pallisera y Fullana (2012), están de acuerdo en que el proceso de transición a la vida adulta y laboral de los jóvenes con DFI requiere de una acción de orientación en la etapa secundaria, con énfasis intencional en la promoción de estrategias optimizadoras de la inclusión socio-laboral. Hablamos de un colectivo que presenta cierta vulnerabilidad, debido a su tradicional ausencia de los contextos sociales a los que hacemos referencia, y que requiere por tanto de una intervención lo más temprana posible, orientada hacia su independencia y autodeterminación en la vida adulta.

Durante los procesos de educación, formación profesional y orientación educativa de los jóvenes con DFI, resulta de gran relevancia el papel que desempeñan los profesionales de los centros educativos. Y es que, tal como refieren Pereda, De Prada y Actis (2003), estos profesionales cuentan con una posición de mediadores entre el poder político, la actividad económica y las familias, una situación que les permite conocer las fortalezas y debilidades de cada uno de estos agentes, diseñando una estrategia de intervención socio-educativa basada en la realidad que acontece a dichos jóvenes. Indudablemente, la intervención directa de los profesionales en el contexto educativo, ya sea desde los niveles de educación secundaria o desde la formación profesional, promueve que estos conozcan de cerca la realidad de la problemática formativa y socio-laboral a la que se enfrentan los jóvenes con DFI, así como las fórmulas de interacción existentes entre estos y los agentes ya mencionados, y las demandas y necesidades que se emiten desde el entorno familiar.

Si hablamos de la inclusión de los jóvenes con DFI en su tránsito a la vida adulta y laboral, debemos tener en cuenta aportaciones como las de Pallisera, Fullana y Vilà (2005), quienes valoran el 
centro ordinario como espacio potencialmente favorecedor de las inserciones laborales. Es decir, las posibilidades de promoción social y laboral durante la vida adulta de la persona con DFI aumentarán si el desarrollo integral de esta se produce en un contexto educativo inclusivo, fomentando el desarrollo de relaciones sociales heterogéneas Minimizando así su posible estigmatización a nivel social.

El destacado papel que juegan los profesionales educativos durante todo el proceso educativo y formativo-laboral, viene justificado de la mano del Artículo 5.2 del RD 127/2014 de la LOMCE, el cual expone que "los criterios pedagógicos se adaptarán a las características específicas de los alumnos y las alumnas y fomentarán el trabajo en equipo. Asimismo, la tutoría y la orientación profesional tendrán especial consideración" (Boletín Oficial del Estado, 2014, Sec. I, p. 20157). Es indiscutible la esencial labor que desempeñan los orientadores de los centros educativos y los tutores de cada grupo-clase en los procesos que mencionamos. Estamos hablando de un alumnado heterogéneo, que presenta diferentes necesidades, tanto educativas como sociales, por lo cual, tanto las tutorías individuales y grupales como la orientación profesional deben ir encauzadas a satisfacer las mismas, abordando en todo momento la impartición de aprendizajes transversales a los contenidos puramente académicos. De esta forma, en los procesos a los que hacemos referencia, los profesionales de la acción tutorial y la orientación deben asumir como objetivos principales la atención a la diversidad y la transferibilidad de los aprendizajes adquiridos por el alumno hacia las exigencias impuestas por el mercado laboral.

Centrando nuestra atención en el ámbito de los programas de FPB, detectamos que uno de los principios de la atención integral a su alumnado radica en la colaboración de todo el profesorado, tal como se define en la Orden de 9 de junio de 2015.

La persona que ejerza la tutoría deberá coordinar la relación entre los departamentos de familia profesional que pudieran existir, el Departamento de Orientación, el profesorado que imparte docencia en el grupo y la persona responsable del seguimiento del módulo profesional de Formación en centros de trabajo. (CECD, Boletín Oficial de la Junta de Andaluacía, 2015, Art. 8.5., p. 6).

Como podemos observar, el papel del tutor del grupo-clase adquiere gran relevancia, en tanto que permite que los itinerarios educativos por implantar establezcan una coherencia práctica entre los contenidos de las diferentes materias, con el objeto de proporcionar a los jóvenes con DFI una formación profesional de calidad. Por lo tanto, de acuerdo con la LOMCE (2013), la figura del tutor resulta clave para la coordinación del trabajo de todos los profesionales. Esta idea se justifica en la cercanía y el trato directo y continuado que este profesional tiene con sus alumnos, lo cual le convierte en una fuente esencial de conocimiento e información acerca de las capacidades y dificultades que presenta cada uno de ellos.

Sin embargo, no podemos olvidar el indispensable rol que desempeña el orientador del centro educativo durante todo el proceso relativo a la planificación, diseño y ejecución de la carrera académica y profesional del joven con DFI. Por un lado, debemos reconocer las competencias y habilidades que poseen estos profesionales relativas al ámbito de la orientación educativa y profesional (Figuera, Rodríguez \& Llanes, 2015). Esto es, hablamos de profesionales que cuentan con amplios conocimientos 
sobre el contexto formativo y el socio-laboral, así como con una buena disposición y aptitud hacia el asesoramiento, y una amplia capacidad para vincular las competencias profesionales con las exigencias del mercado de trabajo, las cuales resultan apremiantes en los momentos de incertidumbre y cambio social y laboral que acontecen al alumnado de estas etapas educativas.

Por otro lado, atendiendo a las afirmaciones de Cutanda y González (2015), el papel del Departamento de Orientación además resulta esencial para el desarrollo del trabajo cooperativo, y al mismo tiempo, para mejorar las dificultades de coordinación que pueden surgir entre el profesorado implicado en la impartición de los módulos profesionales de estos programas y en los de competencias básicas, una coordinación, según Maguilot, a todas luces necesaria (2010 como se citó en Cutanda \& González, 2015). El éxito de la intervención educativa debe obedecer, por tanto, a una responsabilidad compartida basada en la comunicación y el trabajo en equipo, y no en actuaciones individuales y desconectadas.

Respecto a la actitud del profesorado encargado de proporcionar a los jóvenes con DFI una educación de calidad que les permita desarrollarse en nuestra sociedad de manera inclusiva, debemos reflejar la importancia de que estos se muestren lo suficientemente concienciados y responsabilizados hacia la inserción laboral de estos jóvenes, lo cual supone para Pallisera (2010) un ámbito para trabajar tanto desde la formación inicial, como en las acciones de formación permanente dirigidas a profesionales. Sabemos que este colectivo tradicionalmente ha sido excluido de los contextos económicos y sociales, y que además el actual empeoramiento del tejido productivo afecta especialmente a los colectivos más vulnerables, como es el caso de las personas con DFI, (Comité Español de Representantes de Personas con Discapacidad, 2009 como se citó en Vilà, Pallisera \& Fullana, 2012), lo cual podría repercutir negativamente en sus deseos y expectativas de vida.

En esta misma línea, tal como apunta Pallisera (2010), las dudas sobre las posibilidades de las personas con diversidad funcional para su inclusión social y laboral afectan también a los profesionales, y con ello condicionan las oportunidades reales de inclusión de estos. Es por esta razón que, para evitar el rechazo y la falta de confianza de nuestra sociedad y de los empresarios, es preciso que los profesionales de la acción formativa y de inserción laboral se muestren comprometidos hacia esta causa y crean firmemente en las potencialidades de los jóvenes con los que intervienen. Así, la práctica de los educadores debe regirse por una convicción plena, que a su vez resulte objetiva y realista hacia las oportunidades de los jóvenes con DFI como trabajadores.

Otro de los principios primordiales que debe regir el trabajo de los profesionales de la acción educativa y de formación profesional de los jóvenes con DFI, además de la atención a la diversidad de su alumnado, es la visibilización y escucha activa de los propios estudiantes. A este respecto, Pallisera (2010) indica que es necesario que el joven manifieste sus propios intereses y pueda tomar sus propias decisiones, mientras los docentes deben aceptar que ellos no son quienes mejor saben lo que necesitan los estudiantes, ayudándoles a formular planes de futuro basados en sus capacidades, necesidades e intereses. La investigadora va más allá de la mera adaptación de los contenidos y apuesta por una colaboración activa entre los docentes y el alumnado, confiando en que los profesionales ofrezcan a los jóvenes con DFI un aprendizaje basado en la participación social y la autodeterminación. 
Analizando con más detalle las ideas de Pallisera, se trata de incitar una evolución en el rol de los profesionales educativos y formativo-escolares, despertando una actitud activa en el joven con DFI.

“(..) recíproca y flexible, donde ambas partes entren en un proceso activo de intercambio con vistas a un fin que no debería ser otro -a medio o largo plazo- que la capacidad de autonomía del sujeto $y$, por tanto la no dependencia institucional" (Pereda et al., 2003, p. 149)

Dicho de otro modo, los profesionales educativos promueven un aprendizaje activo, basado en la experiencia, donde se tienen en cuenta los ritmos de maduración y las habilidades personales del alumno, convirtiéndose en guías y orientadores. Bajo esta metodología, juegan un papel fundamental el diálogo y la escucha activa, que despertarán el sentido crítico y la capacidad de elección y de resolución de conflictos de sus alumnos con DFI.

Teniendo en cuenta los planteamientos expuestos anteriormente, se hace necesario un cambio en la metodología del aula, desde la cual se deben sustituir los modelos tradicionales de enseñanza por nuevas estrategias basadas en los conocimientos y capacidades con los que cuenta el alumnado. En definitiva, tal como expone Barrio (2009), la metodología debe perseguir los objetivos de un currículum común, en el que primen las competencias sobre los contenidos, entendiendo además que el aprendizaje debe estar centrado en el aula, partiendo para ello de las características de todo el alumnado. Para alcanzar estos objetivos, los profesionales deben recurrir a la implantación de metodologías de carácter colaborativo, donde el aprendizaje se produzca de manera globalizada y no parcializada, con el objetivo de preparar a los jóvenes con DFI para su acceso a la vida adulta y laboral. No obstante, para ello es preciso que el trabajo de todo el equipo docente se realice de manera interdisciplinar, contribuyendo así al desarrollo autónomo del alumno, así como a la adquisición por parte de este de habilidades y competencias de índole laboral y social.

Por último, nos gustaría reflexionar acerca de la formación recibida por los profesionales implicados en los contextos educativos y formativo-escolares en los que se encuentran los jóvenes con DFI. A este respecto, Vilà et al. (2012) refieren que, para avanzar en la buena ejecución de las prácticas educativas, hay que incluir estas en los procesos de formación de los profesionales educativos, tomando como objetivo principal que el joven con DFI asuma un papel activo desde el itinerario educativo que debe llevarle a la inclusión socio-laboral. Es decir, la formación de los docentes debe estar basada en las propias prácticas educativas, difundiendo así un conocimiento más acertado de la realidad del sistema educativo actual y de las dificultades a las que tienen que hacer frente los jóvenes con DFI para alcanzar la inclusión a nivel educativo, social y laboral, enseñándoles a asumir el rol de guía durante el proceso de aprendizaje

Roselló \& Vergés (2008 como se citó en Pallisera, 2010) reflexionan sobre las debilidades del proceso que mencionamos, que aparecen como consecuencia de la dispersión de los servicios de orientación para el empleo y de la falta de información de los profesionales de la educación sobre ellos. Como consecuencia de esta situación se genera una barrera hacia el conocimiento de los recursos y posibilidades existentes para las personas con DFI, limitando de este modo las acciones formativas y orientativas dirigidas a estos alumnos, que desde los servicios sociales suelen ser canalizados hacia 
servicios especializados. Resulta necesario a este fin, proporcionar a los profesionales del sistema educativo, a través de la formación permanente, un conocimiento exhaustivo de los recursos sociales y comunitarios del área de referencia del centro escolar, que les permita establecer una relación constante con los mismos, a fin de tener una información pormenorizada y realista de la situación del mercado laboral y de las posibilidades reales de los jóvenes con DFI dentro del mismo. Con dicha información, a continuación se deberían reencauzar las acciones educativas del sistema educativo, a fin de instituir una continuidad real entre los aprendizajes proporcionados desde ambos servicios y las demandas existentes dentro del mercado de trabajo.

\section{MÉTODO \\ Diseño}

En el diseño de la investigación hemos optado por la elaboración de un modelo mixto basado en la utilización simultánea de las metodologías cuantitativa y cualitativa, con igualdad de estatus, lo cual nos ofrece una mejor evidencia y comprensión de los fenómenos estudiados, facilitando el fortalecimiento de los conocimientos teóricos y prácticos y ampliando nuestra comprensión sobre el objeto de estudio.

En la vertiente cualitativa de nuestra investigación, hemos utilizado un diseño interpretativo, decantándonos por la estrategia de estudio de casos, que nos va a permitir conocer en profundidad y de manera sistemática, el funcionamiento y configuración del caso específico que hemos seleccionado, observando de modo naturalista los sistemas que acontecen dentro del mismo (Martínez, 1988).

\section{Contexto}

El objeto de nuestro estudio cualitativo son los programas de PCPI de Necesidades Educativas Especiales (NEE) ${ }^{1}$ del IES Concepción Villalba, un centro público de Educación Secundaria de la provincia de Málaga, que cuenta con numerosos recursos especializados para atender a la diversidad de su alumnado durante todas las etapas y programas educativos que oferta. Este centro ordinario acoge a alumnos con NEE en régimen de integración educativa, y cuenta con tres itinerarios específicos de formación profesional desde los que se atiende a este tipo de alumnado, cuyas especialidades son jardinería, marroquinería y cocina. Cada uno de estos itinerarios atiende de 8 a 10 jóvenes con DFI de entre 18 y 23 años.

Estos alumnos cuentan con un nivel de autonomía personal y social suficiente para cursar este tipo de estudios, y son pocos los casos que requieren de una atención asistencial, aunque todos ellos presentan serias carencias a nivel académico. Además, debemos tener en cuenta que estos itinerarios educativos no tienen un carácter obligatorio, por lo que se presupone que pertenecen a familias de un nivel socio-cultural medio, con predisposición a fomentar la inclusión de sus hijos e hijas.

\section{Participantes}

Requeríamos que los participantes de nuestra investigación cuantitativa fueran profesionales del ámbito educativo, que desempeñen su labor en diversos centros de Educación Secundaria de la provincia 
de Málaga, desde los cuales se atiende a las necesidades de los alumnos con DFI, interesándonos especialmente por aquellos que cuentan con programas de formación profesional. Por tanto, seleccionamos una muestra de tipo no probabilístico, por conveniencia. Tras negociar la colaboración de aquellos centros educativos que consideramos que cumplían con los requisitos que exponemos, un total de seis, se solicitó la participación voluntaria de los profesionales de cada centro, que rellenaron el cuestionario de manera individual.

Una vez finalizado el proceso de muestreo, obtuvimos un total de 38 participantes en nuestra investigación, de los cuales un $18.40 \%$ son profesores(as) de Secundaria, un $36.80 \%$ son profesores(as) de Formación Profesional, un $13.20 \%$ son orientadores(as), mientras un $28.90 \%$ son profesionales de apoyo (PT, logopedas, AL, etc.), y por último, existe un $2.60 \%$ de especialistas que se identifican con otros tipos de profesiones. En cuanto a la formación con la que cuentan estos profesionales, un 16.20 $\%$ de ellos son psicólogos(as), un $16.20 \%$ son psicopedagogos(as), un $13.50 \%$ son pedagogos(as), un 5.40 \% son logopedas, un 13.50 \% poseen formación de maestros(as) de Educación Especial, un 32.40 \% son licenciados en alguna materia especializada, son ingenieros o poseen una titulación de CFGS y, por último, el $2.70 \%$ restante posee estudios de otro tipo.

\section{Instrumentos de recolección de datos}

En la parte cuantitativa de nuestro estudio de investigación hemos elaborado un cuestionario, con el cual pretendemos indagar en las valoraciones y percepciones que los profesionales del ámbito educativo tienen sobre los aspectos que intervienen en la educación secundaria y la formación profesional de los jóvenes con DFI tomando como referencia su experiencia personal y profesional. El cuestionario consta de 30 ítems formulados en Escala Likert. Además, está dividido en dos partes; la primera parte consta de seis cuestiones relativas a los datos personales de los participantes, mientras en la segunda parte se realizan 24 cuestiones acerca de los aspectos educativos y formativos.

Para la parte cualitativa de esta investigación hemos realizado un total de nueve entrevistas, individuales y semiestructuradas: a la orientadora del centro, a una de las maestras de apoyo a la integración, a tres profesores de los diferentes talleres de PCPI, a dos alumnas con DFI y a sus familias. Dadas las diferencias existentes entre nuestros entrevistados, diseñamos tres modelos de guiones de preguntas: uno para los profesionales, otro para las familias y otro para los jóvenes alumnos de PCPI de NEE.

\section{Procedimiento}

Como hemos visto, nuestra investigación se basa en la complementariedad de las metodologías cuantitativa y cualitativa, utilizando posteriormente una estrategia de triangulación de datos para el análisis de resultados.

Respecto a la parte cuantitativa de nuestra investigación, una vez finalizada la implantación de nuestro cuestionario, hemos procedido al tratamiento de los datos obtenidos a través del programa estadístico SPSS 12.0, para más adelante proceder al análisis y valoración de los mismos. Una vez obtuvimos las tablas de frecuencia descriptiva de cada una de las cuestiones formuladas, procedimos a su presentación en gráficos con el programa Microsoft Excel 97-2003, para finalizar con la exposición 
de los mismos con su correspondiente descripción y análisis de los resultados obtenidos en cada una de las preguntas formuladas en el cuestionario.

Para facilitar el proceso de triangulación de los datos obtenidos en la parte cualitativa de nuestro estudio hemos realizado un proceso de categorización de la información, que nos permite exponer de manera simplificada las relaciones y conexiones que hemos llegado a estipular entre los elementos y agentes que han emergido de la misma. Con la ayuda del programa informático Nudist Vivo 8.0 hemos establecido 10 categorías. Gracias a estas categorías podemos hacer una catalogación, codificación y clasificación de la información obtenida a través de los instrumentos utilizados, que posteriormente va a contribuir a reseñar, exponer y analizar de manera descriptiva y reflexiva, los datos obtenidos (Rodríguez, Gil \& García, 1996).

\section{RESULTADOS}

A continuación exponemos y analizamos algunos de los datos más relevantes obtenidos a partir de nuestro estudio de investigación, deteniéndonos especialmente en reflexionar acerca del papel que desempeñan los profesionales del ámbito educativo como agentes para la capacitación socio-laboral de los jóvenes con DFI. En primer lugar conoceremos los resultados de nuestro estudio estadístico.

Para empezar, queremos averiguar si los profesionales del ámbito educativo están de acuerdo en que la formación de los docentes de los centros educativos es satisfactoria para capacitar a los alumnos con DFI para conseguir y mantener un empleo. Los datos obtenidos nos muestran que el $38.90 \%$ de estos cree que sí, porque son conscientes de la necesidad de mejorar la empleabilidad de este colectivo, mientras un 8.30 \% corrobora que sí, aunque no la llevan a la práctica y, por último, un $52.80 \%$ refiere que no, y es un tipo de formación que se debería potenciar.

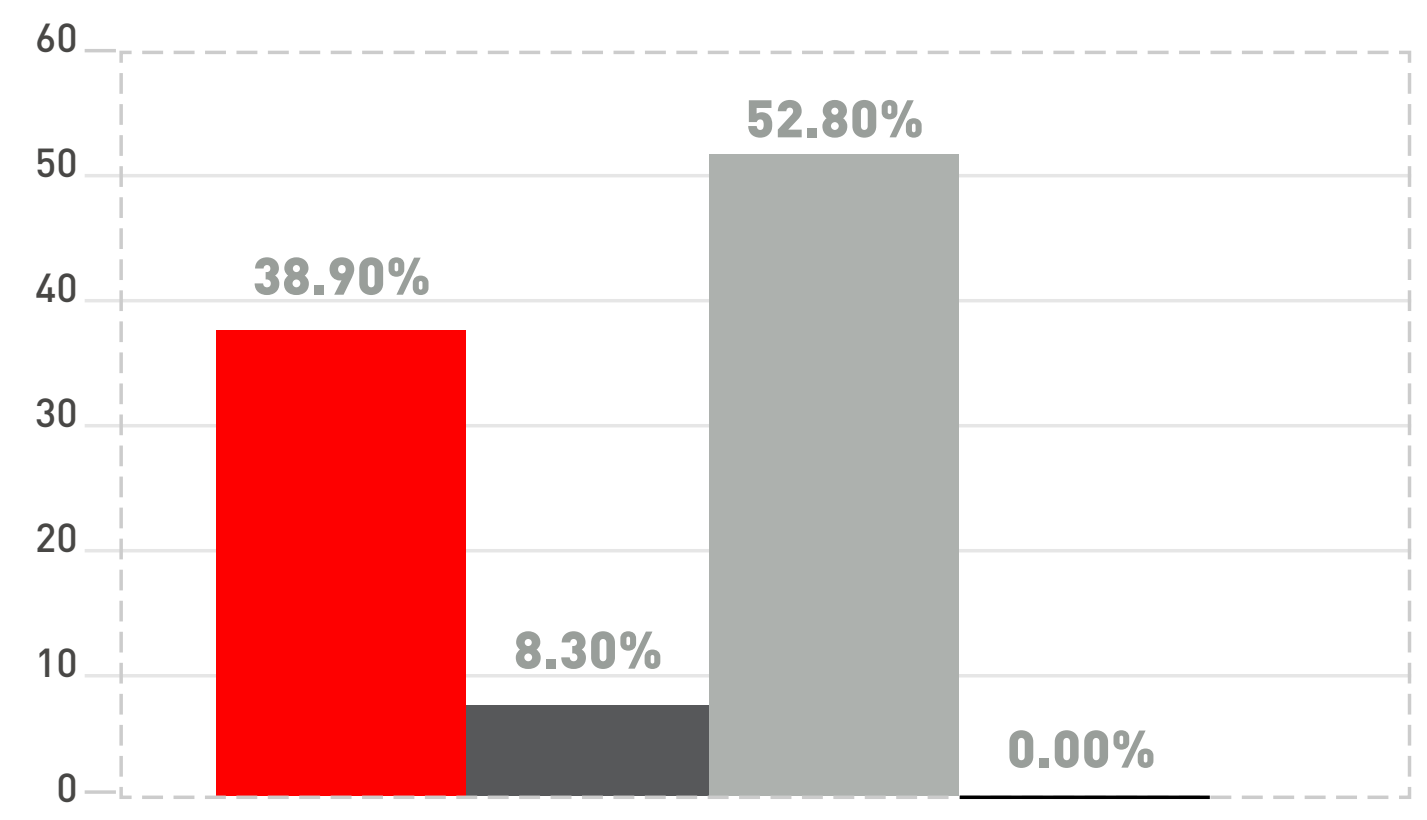

Sí, porque son conscientes de la necesidad de mejorar la emplealidad del este colectivo

Sí, aunque no la llevan a la práctica

No, y es un tipo de formación que se debería potenciar

No, porque no les parece relevante preoparar a estos jóvenes para adquirir un empleo

Figura 1. La formación de los docentes de los centros educativos es satisfactoria para capacitar a los alumnos con diversidad funcional intelectual para conseguir y mantener un empleo. 
Como podemos observar, más de la mitad de los profesionales del ámbito formativo-escolar que participaron en nuestro estudio se muestra de acuerdo en que la formación de los docentes de los centros educativos no es satisfactoria para capacitar a los alumnos con DFI para conseguir y mantener un empleo, pero es un tipo de formación que se debería potenciar. Por el contrario, este grupo va precedido de un amplio volumen de profesionales que piensa que los docentes sí cuentan con dicha formación, porque son conscientes de la necesidad de mejorar la empleabilidad de este colectivo, existiendo además un último grupo que también valora positivamente la formación de los docentes de los centros educativos al fin anteriormente expuesto, aunque no la llevan a la práctica.

De los datos obtenidos podemos deducir que, según el punto de vista de los profesionales, desde el propio sistema educativo, tanto en las universidades como en otros itinerarios de formación profesional dirigidos a los docentes, posiblemente se desestima la necesidad de preparar a los jóvenes con DFI para su acceso al mercado laboral, legitimando desde las propias instituciones educativas la vulnerabilidad de este colectivo. Esta situación posiblemente ha contribuido a legitimar la discriminación y segregación de los jóvenes con DFI desde el propio sistema educativo, olvidando sus necesidades formativas desde el momento de la formación de los profesionales que intervienen en su educación

Nos gustaría señalar, por todo ello, la necesidad de reconducir la capacitación laboral de los docentes de educación secundaria y formación profesional que se encargan de atender al alumnado con DFI. Para conseguir este objetivo, resulta necesario proporcionar a estos profesionales una formación continua especializada, que contribuya a que estos desarrollen competencias y habilidades para atender a la diversidad de su alumnado, respondiendo al mismo tiempo a las demandas del mercado laboral.

Las temáticas por abordar en este tipo de formación podrían estar relacionadas con la adquisición de metodologías de aprendizaje innovadoras, las estrategias de trabajo interdisciplinar con el resto de profesionales, o el uso de las nuevas tecnologías dentro del aula. Otros posibles ámbitos de formación susceptibles de cursar por los docentes son las estrategias de búsqueda de empleo, emprendimiento e innovación, el conocimiento de las diferentes instituciones sociales de apoyo a la inclusión socio-laboral que existen en el contexto donde se desenvuelven estos jóvenes y la organización de las empresas sociales. Las posibilidades son infinitas, siempre que permitan acercar los aprendizajes académicos a la realidad acontecida en el mercado laboral actual y que despierten en los docentes la concienciación y sensibilización necesarias para apoyar a estos jóvenes en su proceso educativo y de capacitación socio-laboral de manera constructiva y eficaz.

A continuación, hemos indagado en la opinión de los profesionales del ámbito formativo-escolar sobre si la formación de los orientadores de los centros educativos es satisfactoria para guiar y asesorar a los alumnos con DFI a conseguir y mantener un empleo acorde a su perfil. A esta cuestión, un $67.60 \%$ responde que sí, porque son conscientes de la necesidad de mejorar la empleabilidad de este colectivo, mientras un $13.50 \%$ también reivindica que sí, aunque no la llevan a la práctica. Además, existe un $18.90 \%$ de profesionales que piensa que la formación de los orientadores dirigida a este fin no es satisfactoria y es un tipo de formación que se debería potenciar. 


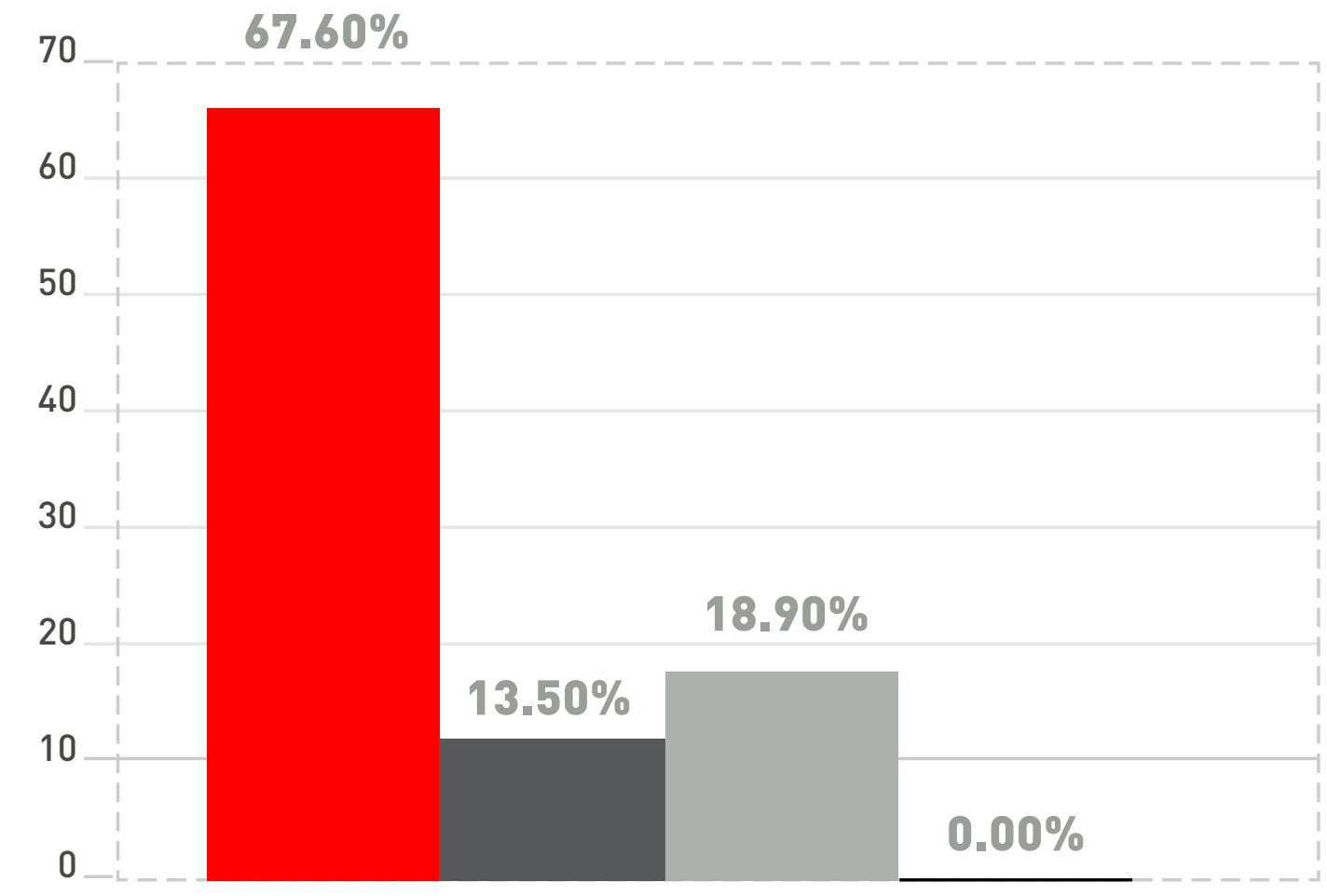

Sí, porque son conscientes de la necesidad de mejorar la emplealidad del este colectivo

- Sí, aunque no la llevan a la práctica

No, y es un tipo de formación que se debería potenciar

- No, porque no les parece relevante preoparar a estos jóvenes para adquirir un empleo

Figura 2. La formación de los orientadores de los centros educativos es satisfactoria para guiar y asesorar a los alumnos con diversidad funcional intelectual a conseguir y mantener un empleo acorde a su perfil.

Los profesionales del ámbito educativo reflejan una mirada positiva hacia la formación de los orientadores de los centros educativos al afirmar que esta sí es satisfactoria para guiar y asesorar a los alumnos con DFI a conseguir y mantener un empleo acorde a su perfil, porque estos son conscientes de la necesidad de mejorar la empleabilidad de este colectivo. Son muy pocos los que comparten sus opiniones entre el resto de las opciones planteadas.

Estas ideas nos dejan entrever una concepción positiva sobre la formación de los orientadores, en contraposición a la que los participantes de nuestro estudio manifestaban con relación a la formación de los docentes. Y es que los orientadores de los centros educativos reciben una formación especializada en la orientación profesional del alumnado en general. Además, estos profesionales se encuentran especializados en materias como la psicología, la pedagogía o la psicopedagogía, por lo cual se presupone que tienen un mayor conocimiento sobre las dificultades personales, sociales y laborales a las que tiene que enfrentarse el alumnado con DFI. Al mismo tiempo, su formación les ha permitido adquirir diversas habilidades y competencias, que mejorarán su intervención a la hora de responder a las necesidades y demandas de este tipo de alumnado.

Por consiguiente, en base a estas dos últimas cuestiones valoramos como necesario realizar un trabajo interdisciplinar por parte de los profesionales donde, a través de un seguimiento individualizado de los alumnos, los orientadores apoyen la labor de los docentes para promover dentro del aula un aprendizaje transversal en competencias laborales y sociales. En definitiva, pensamos que la labor del orientador escolar resulta fundamental durante los procesos de orientación profesional, formación e inserción laboral, especialmente en el caso de los jóvenes con DFI. Por todo ello, dada su relevancia, 
consideramos que la del orientador escolar es una figura escasamente reconocida a nivel social, ya que generalmente, e incluso desde la propia comunidad educativa, se olvida en muchas ocasiones su potencial humano y profesional, dejando todo el peso de la intervención en manos del equipo docente.

Por último, si observamos los resultados obtenidos relacionados con la afirmación que plantea que los profesionales del centro colaboran de manera interdisciplinar para dar una educación de calidad a los jóvenes con DFI, observamos que un 27.80 \% de los profesionales educativos piensa que sí, siempre. A su vez, existe un $61.10 \%$ que está de acuerdo en que sí, en algunas ocasiones los profesionales piden asesoramiento al personal de apoyo (PT, logopeda, orientador/a, etc.), además de un $8.30 \%$ de estos profesionales que señala que no, pues resulta imposible por tiempo, espacios, etc. Por último, un $2.80 \%$ de los participantes responde que no, cada profesional se limita a impartir su área de aprendizaje.

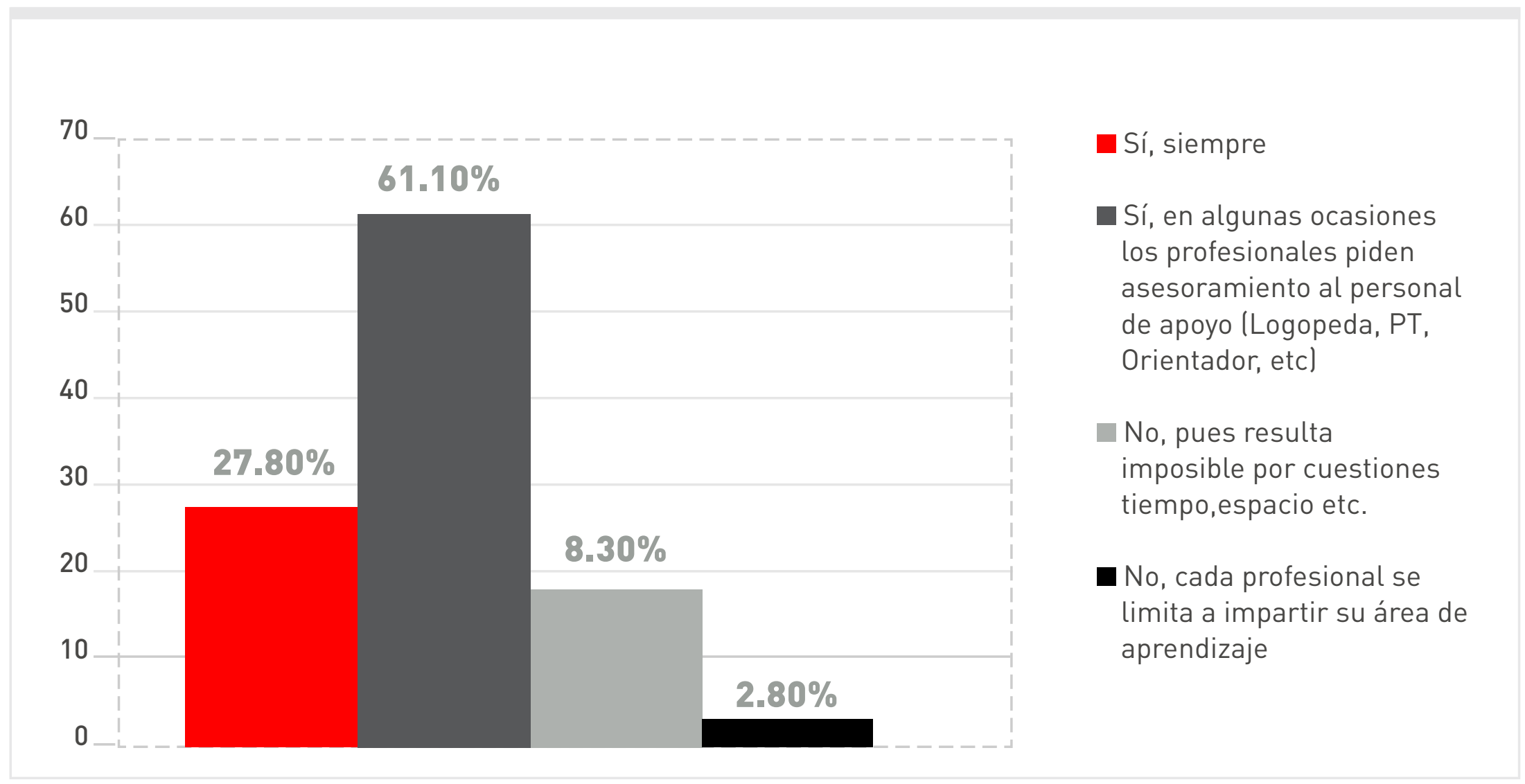

Figura 3. Los profesionales del centro colaboran de manera interdisciplinar para dar una educación de calidad a los jóvenes con diversidad funcional intelectual.

De esta manera, somos conscientes de que los participantes de nuestro estudio determinan de manera generalizada que los profesionales del centro sí colaboran de manera interdisciplinar para dar una educación de calidad a los jóvenes con DFI, primando los que opinan que sí, en algunas ocasiones los profesionales piden asesoramiento al personal de apoyo (logopeda, PT, orientador/a, etc.), sobre otro amplio grupo de especialistas que también piensan que sí, siempre los profesionales colaboran de manera interdisciplinar.

Ofrecer una atención educativa interdisciplinar resulta primordial en el desarrollo cognitivo, social y laboral de los jóvenes con DFI. Y es que estamos hablando de un tipo de alumnado que requiere 
de una estimulación constante, adaptada a sus necesidades y a sus ritmos de maduración, que debe permitirles adquirir las capacidades y habilidades educativas y formativo-laborales necesarias para desenvolverse en los diferentes contextos de nuestra sociedad durante su transición a la vida adulta. Gracias al trabajo interdisciplinar de todo el equipo docente, se evita la impartición de contenidos de manera inconexa y parcializada y se produce una evolución global en las competencias de este tipo de alumnado.

La problemática de ofrecer una intervención interdisciplinar a los alumnos con DFI surge de la falta de tiempo o de una formación especializada, lo cual provoca que los docentes en muchas ocasiones decidan delegar la intervención educativa del alumnado con NEE en los profesionales especializados, por lo que estos alumnos únicamente se encuentran atendidos en los momentos en que salen a trabajar al aula de apoyo a la integración. Esta situación tiene como consecuencia una posible desatención educativa dentro del aula ordinaria, así como cierto aislamiento social del resto de sus compañeros. Considerando esta situación, debemos reseñar que estas malas prácticas docentes nada favorecen la inclusión educativa de los alumnos con DFI, perjudicando su aprendizaje y socialización, por la descontextualización y falta de continuidad de las actividades por realizar. Una posible solución a este conflicto radica en la implicación y participación real del personal de apoyo a la integración en la rutina normal del aula y, de manera paralela, se hace necesario dotar a los docentes de una adecuada formación que les permita atender a las necesidades de todos sus alumnos, teniendo como objetivo establecer una misma línea de trabajo y fijar unos objetivos educativos concretos.

Llegados a este punto, vamos a exponer y analizar las evidencias que hemos extraído acerca del tema que nos ocupa de las entrevistas realizadas en nuestro estudio de caso. Así, comenzaremos conociendo la opinión del profesor de taller de PCPI de la especialidad de cocina, sobre la atención a las necesidades de los jóvenes con DFI por parte del equipo docente.

Estamos hablando de que podemos ser ciento y pico profesores, pues ya estamos hablando de que 20 o 25 son solo para ellos. Claro que, evidentemente, necesitan más recursos que los demás, porque el profesor de filosofía llega, pone el tema en la pizarra y luego hace el examen; si está bien, aprobado y si está mal, suspenso. Pero con ellos no puedes actuar así: ellos necesitan otro tipo de atención, otro tipo de sensibilidad y trabajar de otra manera muy distinta. (Entrevista a José, profesor de taller de PCPI Cocina, pp. 12- 13).

Este profesor manifiesta la necesidad de destinar unos recursos humanos especializados en la atención a las particularidades de aquellos jóvenes que presentan NEE. Estamos hablando de una escuela en la que el conocimiento se imparte de manera parcelada y las metodologías empleadas dentro del aula apuestan por la homogeneización del alumnado, lo cual impide que los profesores puedan atender a estos alumnos de manera adecuada.

En nuestra opinión, la labor desempeñada por los profesionales de apoyo a la integración, como los PT, logopedas o monitores, es una parte fundamental en la evolución educativa y socio-laboral de estos jóvenes. De la misma forma, debemos ser conscientes de las limitaciones de todo el personal, dado que no pueden atender a cada uno de los alumnos que presenta NEE durante toda la jornada escolar, 
y además su atención depende en gran parte de la disponibilidad física de otros espacios del centro. Como consecuencia de este hecho, la inclusión de estos alumnos se ve dificultada al no compartir un mismo espacio de aprendizaje con el resto de sus compañeros durante un tiempo considerable de su horario escolar, lo cual perjudica su socialización. Durante el tiempo que permanecen dentro del aula no pueden participar activamente en las actividades que realiza el resto del grupo, lo cual perjudica aún más su inclusión socio-educativa.

Este docente además alude a la sensibilidad del profesorado para atender a los jóvenes con DFI. Pensamos que no solo el personal de apoyo a la integración, sino todo el equipo docente, debe estar concienciado y sensibilizado hacia las diferencias estos jóvenes, a fin de proporcionarles una educación de calidad, así como de evitar situaciones de rechazo y discriminación con su grupo de iguales.

A continuación, vamos a conocer la opinión los padres de una alumna de uno de los talleres de PCPI, sobre la atención a la diversidad de este tipo de alumnado.

Entrevistadora: Y los profesores muchas veces tampoco saben qué hacer.

Carmen: Ni pueden, ni saben ni pueden. Es que no pueden porque tienen que atender a otros; tendría que haber una persona atendiendo a aquellas personas que tengan unas NEE. ¿Qué hace, deja al resto de la clase? Y si atiende al resto de la clase, ¿qué pasa? Que siempre, lo lógico es que atienda a 20 y no atienda a 3, entonces aquellos 3 están sentados en una silla en un rincón. (Entrevista a padres de alumna, p. 14).

Esta madre señala la gran dificultad a la que deben enfrentarse los docentes que cuentan con varios alumnos con NEE dentro de su aula, y no cuentan con las estrategias metodológicas y el tiempo suficiente para atender a todo su alumnado. Si repetimos una de las frases pronunciadas por esta madre "los profesores ni saben ni pueden", observamos que, en su opinión, el origen de este problema se fundamenta en la ausencia de apoyos al profesorado dentro del aula para que puedan atender de manera eficaz a todos los alumnos sin tener que elegir a quién dedicar sus atenciones

Realmente todos los alumnos cuentan con capacidades y necesidades educativas diferentes, no solo aquellos que tienen NEE, por lo cual pensamos que es necesario dejar atrás la tradicional concepción de la educación homogeneizadora con una metodología de carácter magistral. Si bien es cierto que los jóvenes con DFI, en ocasiones pueden requerir de la presencia de apoyos específicos que faciliten sus aprendizajes y resuelvan las dificultades de adaptación educativa y social que puedan tener. Por esta razón, estamos de acuerdo en que es necesaria la presencia de algún profesional de apoyo a la integración, que favorezca la interacción de los jóvenes con DFI en la dinámica ordinaria del aula.

Por otra parte, el desconocimiento por parte de los docentes de estrategias individualizadoras de aprendizaje que respeten la heterogeneidad de este y de todo el alumnado, legitiman las situaciones de desatención y aislamiento de las que nos habla Carmen. En definitiva, para que los docentes puedan atender a todos los alumnos es necesaria la utilización de metodologías de aprendizaje cooperativo, que apuesten por el respeto y la atención a la diversidad del alumnado, y en las que primen las competencias sobre los contenidos. 
Por último, la maestra en Pedagogía Terapéutica (TP) del centro, nos expone su visión sobre los recursos materiales con los que cuenta desde el centro educativo, y sobre cuál es su compromiso con la educación y formación de sus alumnos.

El año que estuve trabajando de AL tenía que ir a las tiendas de los chinos y cargarme de cosas porque yo necesito para trabajar globos, pomperos, silbatos y cosas de esas, y si no hay, no puedo trabajar. Y tenía que comprarlo yo, pero claro, eso te lo puedes permitir, gastarte al mes $20 €$ en materiales, pero en ordenadores ya no puedo (Entrevista a Rocío, maestra PT).

Como podemos observar, esta maestra reconoce que no cuenta con los recursos materiales que necesita para desempeñar su actividad profesional con eficacia y rigor. Para evitar un estancamiento en la evolución socio-educativa de sus alumnos con NEE y poder proporcionarles una atención de calidad, esta profesional se ve en la obligación de aportar sus propios recursos. Dada la situación, decide comprar ella misma aquello que necesita para trabajar, teniendo que invertir un tiempo extra, además de realizar un esfuerzo económico para asumir sus funciones docentes.

El papel de los profesionales de apoyo a la integración, como hemos visto, resulta de gran importancia para el desarrollo personal, educativo y social de los alumnos con DFI, ya que cuentan con una formación específica en la atención a las dificultades de estos, tales como dificultades en el lenguaje o problemas de aprendizaje. Sin embargo, no basta con dotar a los centros educativos de este tipo de recursos humanos, si estos no cuentan con los medios materiales suficientes para realizar una intervención de calidad. Es responsabilidad de las instituciones educativas proporcionar a estos centros recursos materiales especializados, que contribuyan a mejorar la labor de los profesionales de apoyo a la integración, pues esta carencia de recursos puede provocar cierto malestar docente, y consecuentemente, falta de compromiso y motivación con su labor, por lo cual podría verse mermada o ralentizada la evolución de los alumnos con DFI.

Desde nuestro punto de vista, resulta fundamental que el personal de apoyo a la integración, así como el resto del equipo docente, cuente con los medios materiales y las infraestructuras adecuadas, que les permitan desempeñar su cometido de educar y formar a los jóvenes con DFI para la vida adulta y laboral.

Sin embargo, la comunicación y utilización de otros recursos sociales y comunitarios también podría beneficiar el proceso educativo y formativo escolar de los jóvenes con DFI. Existen numerosas entidades sociales que dirigen sus esfuerzos hacia la inclusión socio-laboral de las personas con DFI, y que cuentan con recursos especializados para atender a su desarrollo socio-laboral. Otorgar a los profesionales de los centros educativos la formación e información necesarias sobre este tipo de recursos, fomentará el trabajo colaborativo con este tipo de instituciones, permitiendo atender con mayor eficacia a las necesidades de este tipo de alumnado y, a su vez, encauzar las acciones educativas y formativas hacia el acceso al empleo y la vida adulta. 


\section{DISCUSIÓN}

Tras la realización de este estudio de investigación, podemos confirmar la idea de considerar la intervención de los profesionales educativos como clave esencial para capacitar a los jóvenes con DFI para una futura inclusión socio-laboral de éxito.

No obstante, hemos sido testigos de las carencias formativas de algunos de estos profesionales en sus funciones de atender a la diversidad de aquel alumnado que presenta NEE. A tal efecto, estamos de acuerdo con Sales, Moliner y Sanchiz (2001) en manifestar la falta de formación coherente y bien planificada tanto inicial como permanente, que hace que el profesorado se sienta desorientado, incapacitado, desinteresado e incluso que llegue a rechazar abiertamente la integración de estos alumnos. Por tanto, se hace necesario revisar los planes de estudio de aquellas titulaciones susceptibles de desempeñar su actividad dentro de los contextos educativos y formativoescolares. En aquellos casos en que no sea posible, la impartición de una formación continua en metodologías de aprendizaje innovadoras, nos resulta una idea eficaz para poder atender con éxito a las particularidades de estos jóvenes. Al mismo tiempo, la concienciación y sensibilización de todos los profesionales que atienden a los jóvenes con DFI, debe ser una medida prioritaria para conseguir la implicación de todos ellos con respecto a la inclusión socio-laboral de estos, la cual se conseguirá a través de charlas y talleres formativos.

A su vez, el trabajo interdisciplinar de orientadores educativos, docentes y personal de apoyo a la integración, optimizará los procesos educativos de este tipo de alumnado. De este trabajo interdisciplinar, con relación a los estudios de Verdugo (2004), se extrae como una de las labores fundamentales que debe asumir todo el equipo docente, una precisa y frecuente evaluación de los apoyos o ayudas individuales que necesita cada alumno. Es decir, la atención educativa de los jóvenes con DFI que realizan estudios secundarios o posobligatorios, podrá verse beneficiada de la comunicación constante entre todos los miembros del claustro, que deberán seguir una misma línea de trabajo y perseguir unos objetivos comunes y bien definidos.

Si hablamos de los objetivos educativos, los profesionales educativos deben velar por otorgarles una intencionalidad clara y práctica hacia la inserción en el mercado laboral. Es decir, el currículum debe centrarse en la adquisición de competencias personales, sociales y laborales, que primen sobre los contenidos académicos y que permitan a los jóvenes con DFI desenvolverse en los contextos propios de la vida adulta. En la adquisición de este tipo de capacidades y habilidades, la utilización por parte del profesorado de metodologías de aprendizaje cooperativo y de las TICS podrían resultar ser herramientas claves, que potenciarán la participación activa de su alumnado, permitiéndoles ser protagonistas de su desarrollo educativo y formativo-laboral y respetando sus diferencias.

Durante todas estas etapas, el orientador escolar juega un rol imprescindible, en tanto que resulta ser un intermediario entre el centro educativo y el mercado laboral. Es decir, el orientador, partiendo de las características personales y laborales del alumnado, y de acuerdo con las demandas del mercado de trabajo, debe realizar una orientación profesional temprana de los alumnos con DFI. Con la ayuda del resto de profesionales deberá diseñar un plan estratégico de formación y de atención socio-educativa, adaptado a las características, necesidades e intereses de cada joven. 
No podemos olvidar tampoco la importancia de implicar a las familias de estos jóvenes durante el transcurso de su educación secundaria y formación profesional y no demorar su colaboración hasta el momento de la inserción laboral (Valls, Vilà \& Pallisera, 2003). Para ello, es necesaria la comunicación constante entre las familias y el equipo docente, que potencie el intercambio de impresiones y percepciones y que facilite el seguimiento de una misma línea de trabajo desde la escuela y el hogar. Una adecuada coordinación entre el trabajo de los profesionales y el de la familia ayudará a favorecer el crecimiento cognitivo, emocional y social del alumno, así como a facilitar su futura inclusión socio-laboral.

Otra de las cuestiones que queremos manifestar es la necesidad de garantizar el bienestar emocional de los profesionales del ámbito educativo, en tanto que estos deben contar con unas condiciones laborales óptimas, que le ayuden a desempeñar su trabajo con la suficiente eficacia, responsabilidad y compromiso. Estos profesionales, por lo general, deben hacer frente a una sobrecarga de trabajo generada por la escasez de recursos humanos suficientes para atender a todo el alumnado. Además, en ocasiones deben extralimitarse en sus funciones para garantizar el éxito educativo de estos, a pesar de no contar con los recursos materiales necesarios para desempeñar su labor con rigor.

Por lo tanto, se hace fundamental fomentar la comunicación y coordinación de estos profesionales con aquellos que desempeñan su labor desde los servicios de formación para el empleo y de inserción laboral que se ofertan desde las entidades sociales dedicadas a apoyar la inclusión de las personas con DFI. Estos servicios, además de contribuir al crecimiento integral de la persona, efectúan el proceso de intermediación con el entorno empresarial y la intervención directa dentro del contexto laboral (Lucas et al., 2005), por lo cual suponen un referente dentro del proceso que referimos., Con este tipo de relaciones se optimizarán la formación y orientación profesional de los alumnos con DFI, en tanto que estos podrán recibir también los apoyos proporcionados desde los recursos de dichas instituciones. La comunicación con este tipo de entidades contribuirá a generar la unión entre los diferentes recursos sociales y comunitarios, mejorando la imagen social de este colectivo y creando nuevas sinergias que facilitarán la futura inclusión socio-laboral de los jóvenes con DFI.

Por último, debemos señalar que después de todo el procedimiento de análisis y reflexión que hemos llevado a cabo en la ejecución de este estudio de investigación, valoramos como imprescindible que, desde la comunidad educativa y la sociedad en general, deba tenerse en cuenta el esfuerzo que estos profesionales realizan, gratificándoles con el reconocimiento social que merecen, poniendo a su disposición los recursos materiales y humanos necesarios para evitar una saturación en la práctica de su actividad profesional, y ofreciéndoles una retribución económica acorde con su esfuerzo e implicación. Esta nueva concepción de la labor desempeñada por estos profesionales, mejorará su situación emocional, social y laboral, lo cual seguro beneficiará la inclusión de los jóvenes con DFI durante su vida escolar y su tránsito a la vida adulta.

La finalización de este estudio nos suscita nuevas cuestiones y nos plantea nuevas inquietudes relativas al objeto de nuestro estudio, que van a determinar nuestras futuras líneas de investigación. Estas se identifican con el conocimiento exhaustivo de los planes de estudio de los profesionales implicados en el proceso educativo y formativo de los jóvenes con DFI, y en la capacitación de los 
mismos para atender a la diversidad de su alumnado, encauzando el proceso de aprendizaje de estos hacia el mercado laboral. Otra futura línea de investigación tiene que ver con la dimensión emocional de los docentes, que actualmente tienen que asumir responsabilidades que se exceden de sus funciones, respondiendo a las necesidades de alumnos que requieren de una serie de atenciones y apoyos especializados. Por último, nos gustaría indagar acerca de la calidad de las intervenciones desempeñadas por el personal especializado en atender a la integración de los mismos desde el centro educativo (logopedas, pedagogos terapeutas, psicopedagogos, etc.).

\section{REFERENCIAS}

Barrio, J. L. (2009). Hacia una educación inclusiva para todos. Revista complutense de educación, 20(1), 13- 31. Recuperado de https://goo. gl/9urVZ2

Boletín Oficial de la Junta de Andalucía (29 de junio de 2015). Orden de 9 de junio de 2015, por la que se regula la ordenación de las enseñanzas de Formación Profesional Básica en Andalucía para los cursos académicos 2014/2015 y 2015/2016, se establece el procedimiento de escolarización para el curso académico 2015/2016 y se desarrollan los currículos correspondientes a veinte títulos profesionales básicos Recuperado de https://goo.gl/WUqX73

Boletín Oficial del Estado (24 de diciembre de 2002). Ley Orgánica 10/2002, de 23 de diciembre, de Calidad de la Educación. Recuperado de https:// goo.gl/9yv6bz

Boletín Oficial del Estado (4 de mayo de 2006). Ley Orgánica 2/2006, de 3 de mayo, de Educación. Recuperado de https://goo.gl/2xkao3

Boletín Oficial del Estado (10 de diciembre de 2013). Ley Orgánica 8/2013, de 9 de diciembre, para la mejora de la calidad educativa. Recuperado de https://goo.gl/yvroKl

Boletín Oficial del Estado (5 de marzo de 2014). Real Decreto 127/2014, de 28 de febrero, por el que se regulan aspectos específicos de la Formación Profesional Básica de las enseñanzas de formación profesional del sistema educativo, se aprueban catorce títulos profesionales básicos, se fijan sus currículos básicos y se modifica el Real Decreto 1850/2009, de 4 de diciembre, sobre expedición de títulos académicos y profesionales correspondientes a las enseñanzas establecidas en la Ley Orgánica 2/2006, de 3 de mayo, de Educación. Recuperado de https:// goo.gl/LaUFVF

Cermi reivindica el término 'personas con discapacidad'. (27 de marzo de 2017). La Opinión de Tenerife. Recuperado de https://goo.gl/Nd9GVR

Cutanda, M. T. \& González, M. T. (2015). La colaboración entre docentes del programa de cualificación profesional inicial: el papel del departamento de orientación. Educatio Siglo XXI, 33(2), 303- 322. doi: http://dx.doi.org/10.6018/j/233261

Figuera, P, Rodríguez, M. L. \& Llanes, J. (2015). Transición y orientación: Interrelaciones, estrategias y recomendaciones desde la investigación. REIRE. Revista d'Innovació i Recerca en Educació, 8(2), 1-17. doi: https://doi.org/10.1344/reire2015.8.2821

Jurado, P., Olmos, P. \& Pérez, A. (2015). Los jóvenes en situación de vulnerabilidad y los programas formativos de transición al mundo del trabajo. Educar, 51(1), 211-224. doi: http://dx.doi.org/10.5565/rev/educar.648

Lucas, S., Arias, B., Ovejero, A., Cruz, F., Retortillo, A. \& Rodríguez, H. (2005). Orientación profesional e inserción sociolaboral de personas con discapacidad intelectual. Revista universitaria de ciencias del trabajo, 6, 393- 414. Recuperado de https://goo.gl/Yavf6K

Martínez, M. (1998). La investigación cualitativa etnográfica en educación: Manual teóricopráctico (3ª ed.). México D.F.: Trillas.

Negri, M. I. (2016). Formación e inserción socio-laboral de jóvenes con Diversidad Funcional Intelectual. Una perspectiva pedagógica inclusiva. Málaga: Universidad de Málaga.

Pallisera, M. (2010). Apoyando proyectos de vida inclusivos. Claves para transformar las prácticas socioeducativas de personas adultas con discapacidad intelectual. Revista de Educación Inclusiva, 3(3), 69- 88. Recuperado de https://goo.gl/M9eFHy

Pallisera, M., Fullana, J. \& Vilà, M. (2005). La inserción laboral de personas con discapacidad. Desarrollo de tres investigaciones acerca de los factores favorecedores de los procesos de inserción. Revista de Investigación Educativa, 23(2), 295- 313. Recuperado de https://goo.gl/qUk61g

Pereda, C., De Prada, M.A. \& Actis, W. (2003). La inserción laboral de las personas con discapacidades. Revista del Ministerio de Trabajo y Asuntos Sociales, 55, 151-156. Recuperado de https://goo.gl/R8ZhkZ 
Plataforma discapacitados rechaza término "personas con diversidad funcional". (20 de mayo de 2016). La Vanguardia Cataluña. Recuperado de https://goo.gl/7iBb8Q

Rodríguez, S. \& Ferreira, M. A. V. (2008). Diversidad funcional: sobre lo normal y lo patológico en torno a la condición social de la dis-capacidad. Recuperado de https://goo.gl/zf6p21

Rodríguez, G., Gil, J. \& García, E. (1996). Métodos de investigación cualitativa. Málaga: Aljibe.

Romañach, J. \& Arnau, S. (2006). Bioética y Diversidad Funcional. Recuperado de https://goo.gl/ER3DKR

Romañach, J. \& Lobato, M. (2007). Diversidad Funcional. Nuevo término para la lucha por la dignidad en la diversidad del ser humano. Recuperado de https://goo.gl/95oebC

Sales, Moliner \& Sanchiz (2001). Actitudes hacia la atención a la diversidad en la formación inicial del profesorado. Revista Electrónica Interuniversitaria de Formación del Profesorado, 4(2). Recuperado de https://goo.gl/Tuva8z

Valls, M. J, Vilà, J. \& Pallisera, M. (2003). La inserción de las personas con discapacidad en el trabajo ordinario: el papel de la familia. Revista de Educación, 334, 99- 118. Recuperado de https://goo.gl/jGSYZ9

Verdugo, M. A. (2004). De la segregación a la inclusión escolar. En Obra Social y Cultura Caja Sur (Eds.), Educar para la vida (pp. 9- 18). Recuperado de https://goo.gl/2bWXz7

Vilà, M., Pallisera, M. \& Fullana, J. (2010). ¿Contribuye a la inclusión laboral la formación que recibe el alumnado con discapacidad intelectual en la ESO? Revista de Educación Inclusiva, 3 (3), 51 - 66. Recuperado de https://goo.gl/7wFAcG

Vilà, M., Pallisera, M. y Fullana, J. (2012). La inclusión laboral de los jóvenes con discapacidad intelectual: Un reto para la orientación psicopedagógica. Revista Española de Orientación y Psicopedagogía, 23(1), 85- 93 Recuperado de https://goo.gl/MBEBAL

(c) Los autores. Este artículo es publicado por la Revista Digital de Investigación en Docencia Universitaria del Área de Institutional Research and Effectiveness de la Dirección de Aseguramiento de la Calidad, Universidad Peruana de Ciencias Aplicadas. Este es un artículo de acceso abierto, distribuido bajo los términos de la LicenciaCreativeCommons Atribución-Compartirlgual 4.0 Internacional.I http://creativecommons.org/licenses/bysa/4.0/I, que permite el uso no comercial, distribución y reproducción en cualquier medio, siempre que la obra original sea debidamente citada. 